\title{
Performance Characteristics and Optimal Analysis of an Energy Selective Electron Refrigerator
}

\author{
Cong $\mathrm{Li}^{1}$, Ruiwen $\mathrm{Li}^{1}$, Xiaoguang Luo ${ }^{1,2}, \mathrm{Li} \mathrm{Ma}^{1}$, Jizhou $\mathrm{He}^{* 1}$ \\ ${ }^{1}$ Department of Physics, Nanchang University, Nanchang 330031, P. R. China \\ ${ }^{2}$ Department of Physics, Southeast University, Nanjing 211189, P. R. China \\ E-mail: hjzhou@ncu.edu.cn
}

\begin{abstract}
In this paper, the energy selective electron (ESE) refrigerator with an ideal energy filter based on resonant tunneling is established. It consists of two infinitely large electron reservoirs with different temperatures and chemical potentials, and electrons can be exchanged between the two reservoirs through the ideal energy filter. According to Landauer formula and the assumption of being coupled tightly with the electron current, the expressions for the heat flux, the cooling rate and the coefficient of performance (COP) are derived analytically. The performance characteristic curves such as the cooling rate versus coefficient of performance, the cooling rate and coefficient of performance versus the position of energy level are plotted by numerical calculation. The optimal regions of the cooling rate and the COP are determined. Moreover, we optimize the cooling rate and the COP respectively with respect to the position of energy level. The influence of the width of energy level on performance of the ESE refrigerator is discussed. Finally, based on the optimization criterion $\chi=\varepsilon \cdot \dot{Q}_{C}$ for refrigerator, i.e. the product of the COP times the cooling rate, the optimal performance of the ESE refrigerator is discussed in detail.
\end{abstract}

Keywords: Electron transport; thermoelectric ESE refrigerator; irreversible thermodynamics.

\section{Introduction}

In recent years, scientists have made a series of important breakthroughs in thermoelectric technology and discovered some new nanostructure thermoelectric materials which exhibit a high quality factor [1-5], such as quantum dot, superlattice, nanowires and so on. These materials have two distinct properties: One is a high density of interfaces which is used to reduce parasitic heat flow carried by the crystal lattice on a length scale comparable to the phonon mean-free path. The other is a reduced dimensionality of the electronic system which achieved by band engineering on the scale of the electron wavelength [6]. Moreover, some scholars have used these materials as the working substances to design various thermoelectric devices, such as thermoelectric or thermionic power generators and refrigerators [7-9], Brownian heat engines and refrigerators [10-16], and so on. Furthermore, it has been shown that Carnot efficiency can be reached for reversible electron transport between two reservoirs at different temperatures and chemical potentials by using a sharply tuned energy filter [10, 12]. A double-barrier resonant tunneling structure has been proposed as a theoretical and experimental observation of near-Carnot efficiency [13-16]. The model of an energy selective electron (ESE) heat engine in one dimensional system was firstly proposed by Humphrey et al. [10] in the form of a reversible quantum Brownian heat engines for electrons. After that, a variety of models of the ESE heat engines and refrigerators were proposed. Based on the finite time thermodynamics, the performance of these heat engines and refrigerators are optimized, and many novel results have been obtained [17-33].

On the basis of the previous works $[10,11]$ in which the influence of the center position and width of resonance energy level on the efficiency and power output of the electron heat engine as well as the coefficient of performance and cooling rate of the electron refrigeration was analyzed respectively at an ideal transmission probability and a Lorentzian transmission probability, we study further the performance of the ESE refrigerator with a single resonance in this paper. The expressions for the heat flux and the COP are derived analytically. Under the 
conditions of maximum cooling rate and maximum COP, the optimal performance characteristics of the refrigerator are analyzed. Furthermore, after introducing the figure of merit as a target function, i.e. the product of the cooling rate and the COP, we obtain the optimal cooling rate and COP at maximum figure of merit. The influence of the temperature ratio $\tau$ and the energy level width on the performance of the refrigerator is discussed. The difference between the present paper and Humphrey's work [11] is mainly the use of different optimal target function. The results obtained here are expected to have significant implications for the design of practical thermoelectric refrigerator.

\section{Model and Theory}

Figure 1 shows the schematic diagram of our ESE refrigerator which consists of two different reservoirs and an energy filter contacting the reservoirs both. The two reservoirs have different temperatures, $T_{C}$ and $T_{H}$, and different chemical potentials, $\mu_{C}$ and $\mu_{H}$. They are thermally insulated from each other, and interact only via the energy filter which transmits or blocks electrons selectively. When the chemical potential of the cold reservoir is higher than that of the hot one (i.e. $T_{C}<T_{H}$ and $\mu_{C}>\mu_{H}$ ), the electrons in the two reservoirs will be exchanged from each other through the energy filter due to the temperature gradient and chemical potential gradient. In Figure $1, E^{\prime}$ is the position of energy level of the energy filter, $\Delta E$ is the width of energy level, and $e V_{0}$ is a bias voltage that is applied to the cold reservoir.

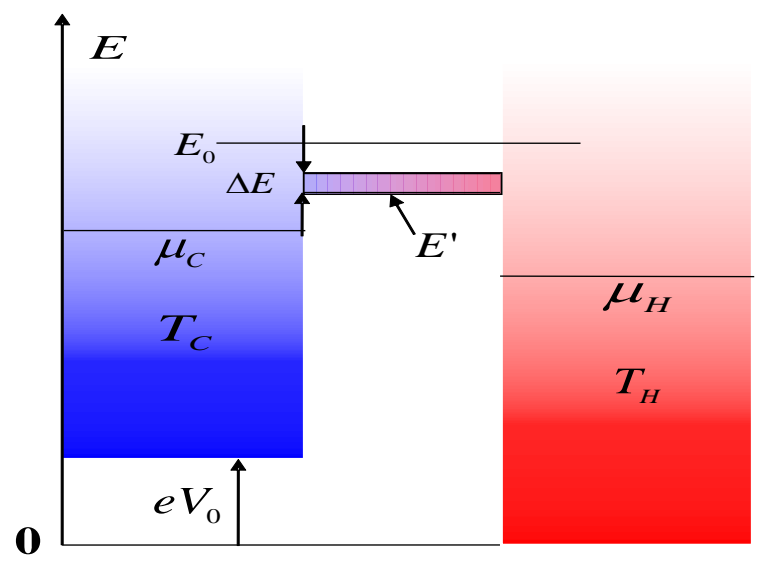

Figure 1. The schematic diagram of an ESE refrigerator.
The average occupation of states of electrons in the reservoirs is described by the Fermi-Dirac function $f=1 /\left\{\exp \left[(E-\mu) / k_{B} T\right]+1\right\}$, where $k_{B}$ is the Boltzmann constant, $E$ is energy of electrons, $\mu$ is chemical potential, $T$ is temperature. When the occupation of states of the transmitted electrons in the two reservoirs are equal (the energy of the electron is marked $E_{0}$ in Figure 1 ), no net electron current occurs, and this special energy level is $E_{0}=\left(\mu_{C} T_{H}-\mu_{H} T_{C}\right) /\left(T_{H}-T_{C}\right)$. Moreover, the net electron current will flow from cold to hot reservoir when $\mu_{C}<E<E_{0}$ (i.e. $f_{C}>f_{H}$ ), and it will reverse when $E>E_{0}$ (i.e. $\left.f_{C}<f_{H}\right)$.

The heat change of a reservoir due to one transmitted electron is given by $E-\mu$. Thus, an electron absorbs the amount of heat $\Delta Q_{C}=E-\mu_{C}$ from cold reservoir when it leaves the cold reservoir, and then releases the amount of heat $\Delta Q_{H}=E-\mu_{H}$ to hot reservoir when it arrives at the hot reservoir, where $e V=\Delta Q_{H}-\Delta Q_{C}=\mu_{C}-\mu_{H}$. As a refrigerator, the net electron current should flow from the cold to the hot reservoirs and the net heat flux out of the cold reservoir should be positive as well. So, for the single level ESE mechanism, the cooling region of the transmitted electrons is $\mu_{C}<E<E_{0}$. For an ideal energy filter with energy width, the transmission probability of the electrons is assumed to be

$\zeta(E)=\left\{\begin{array}{cc}1, & E^{\prime}<E<E^{\prime}+\Delta E \\ 0, & \text { elsewhere }\end{array}\right.$

where the electrons with the energy in $E^{\prime}<E<E^{\prime}+\Delta E$ can be transmitted completely and others are blocked.

According to the Landauer formula the net electron current between the two reservoirs is given by

$I=\frac{2 e}{h} \int_{E^{\prime}}^{E^{\prime}+\Delta E} \varsigma(E)\left(f_{C}-f_{H}\right) d E$

where $e$ is the charge of an electron, $h$ is the Planck constant. The heat flux into the hot reservoir and out of cold reservoir associated with the electron current is given, respectively, by 


$$
\begin{aligned}
& \dot{Q}_{H}=\frac{2}{h} \int_{E^{\prime}}^{E^{\prime}+\Delta E}\left(E-\mu_{H}\right)\left(f_{C}-f_{H}\right) d E \\
& \dot{Q}_{C}=\frac{2}{h} \int_{E^{\prime}}^{E^{\prime}+\Delta E}\left(E-\mu_{C}\right)\left(f_{C}-f_{H}\right) d E
\end{aligned}
$$

where $\dot{Q}_{C}$ is defined as the cooling rate. Subsequently, the power input and the COP are given by

$$
P=\left(\dot{Q}_{H}-\dot{Q}_{C}\right)=\frac{2}{h} \int_{E^{\prime}}^{E^{\prime}+\Delta E}\left(\mu_{C}-\mu_{H}\right)\left(f_{C}-f_{H}\right) d E
$$

$$
\varepsilon=\frac{\dot{Q}_{C}}{P}=\frac{\dot{Q}_{C}}{\left(\dot{Q}_{H}-\dot{Q}_{C}\right)}
$$

With the help of the two integral functions

$$
\begin{aligned}
& \int_{a}^{b} 1 /(1+x) d x=\left.\ln (1+x)\right|_{a} ^{b} \\
& \int_{a}^{b} \ln (x) /(1+x) d x=[\ln (x) \ln (1+x)+\text { PolyLog }(2,-x)]_{a}^{b}
\end{aligned}
$$

the expressions for the cooling rate and the COP are derived analytically as

$$
\begin{aligned}
\dot{Q}_{C} & =\frac{2}{h} k_{B}^{2} T_{C}^{2}\left\{\left[\ln \left(e^{-r_{C}} e^{-\Gamma_{C}}\right) \ln \left(1+e^{-r_{C}} e^{-\Gamma_{C}}\right)+\right.\right. \\
& P \operatorname{olyLog}\left(2,-e^{-r_{C}} e^{-\Gamma_{C}}\right)-\ln \left(e^{-r_{C}}\right) \ln \left(1+e^{-r_{C}}\right) \\
& \left.-\operatorname{PolyLog}\left(2,-e^{-r_{C}}\right)\right]-\frac{1}{\tau^{2}}\left[\ln \left(e^{-r_{H}} e^{-\Gamma_{H}}\right) \ln (1\right. \\
+ & \left.e^{-r_{H}} e^{-\Gamma_{H}}\right)+\operatorname{PolyLog}\left(2,-e^{-r_{H}} e^{-\Gamma_{H}}\right)-\ln \left(e^{-r_{H}}\right) \\
\times & \left.\ln \left(1+e^{-r_{H}}\right)-\operatorname{PolyLog}\left(2,-e^{-r_{H}}\right)\right]-\left(\frac{r_{H}-\tau_{C}}{\tau^{2}}\right) \\
& \left.\times\left[\ln \left(1+e^{-r_{H}} e^{-\Gamma_{H}}\right)-\ln \left(1+e^{-r_{H}}\right)\right]\right\}
\end{aligned}
$$

$$
\begin{aligned}
& \varepsilon=\left\{\left[\ln \left(e^{-r_{C}} e^{-\Gamma_{C}}\right) \ln \left(1+e^{-r_{C}} e^{-\Gamma_{C}}\right)+\operatorname{PolyLog}(2,\right.\right. \\
&\left.-e^{-r_{C}} e^{-\Gamma_{C}}\right)-\ln \left(e^{-r_{C}}\right) \ln \left(1+e^{-r_{C}}\right)-\operatorname{PolyLog}(2, \\
&\left.\left.-e^{-r_{C}}\right)\right]-\frac{1}{\tau^{2}}\left[\ln \left(e^{-r_{H}} e^{-\Gamma_{H}}\right) \ln \left(1+e^{-r_{H}} e^{-\Gamma_{H}}\right)+\right. \\
& P o \log \left(2,-e^{-r_{H}} e^{-\Gamma_{H}}\right)-\ln \left(e^{-r_{H}}\right) \ln \left(1+e^{-r_{H}}\right) \\
&\left.-P o l y \log \left(2,-e^{-r_{H}}\right)\right]-\left(\frac{r_{H}-\pi_{C}}{\tau^{2}}\right)\left[\ln \left(1+e^{-r_{H}} e^{-\Gamma_{H}}\right)\right. \\
&\left.\left.-\ln \left(1+e^{-r_{H}}\right)\right]\right\} \\
& /\left\{\left(\frac{r_{H}}{\tau}-r_{C}\right)\left[\ln \left(\frac{e^{r_{C}}+1}{e^{r_{C}}+e^{-\Gamma_{C}}}\right)-\frac{1}{\tau} \ln \left(\frac{e^{r_{H}}+1}{e^{r_{H}}+e^{-\Gamma_{H}}}\right)\right]\right\}
\end{aligned}
$$

respectively, where $\operatorname{Poly} \log (2,-x)$ is the Nielsen function, $r_{H}=\left(E^{\prime}-\mu_{H}\right) / k_{B} T_{H}$ and $r_{C}=\left(E^{\prime}-\mu_{C}\right) / k_{B} T_{C}$ are two dimensionless scaled energies, $\Gamma_{C}=\Delta E / k_{B} T_{C}$ and $\Gamma_{H}=\Delta E / k_{B} T_{H}$ are two dimensionless widths of energy level, and $\tau=T_{C} / T_{H}$ is the temperature ratio. The cooling rate and the COP are two important performance parameters of the refrigerator. And it is found that they are the functions of the position of energy level $E^{\prime}$, the width of energy level $\Delta E$, the temperatures of $T_{C / H}$, and the chemical potentials of cold/hot reservoirs $\mu_{C / H}$

\section{Performance Characteristics Analysis}

To illustrate the performance characteristics of the ESE refrigerator, the curves of the cooling rate $\dot{Q}_{C}$ and the COP $\varepsilon$ versus the position of energy level $E^{\prime}$ are plotted at different width of energy level $\Delta E$, as shown in Figures 2 and 3. In the numerical calculation, it is fixed that $T_{C}=1 \mathrm{~K}$, $T_{H}=2 \mathrm{~K}, \mu_{C}=12 \mathrm{~J}, \mu_{H}=10 \mathrm{~J}, k_{B}=1 \mathrm{~J} / \mathrm{K}$ and $h=1 \mathrm{~J} \cdot \mathrm{s}$ for convenience.

It is seen from Figure 2 that the cooling rate $\dot{Q}_{C}$ increases firstly and then decreases as the position of energy level $E^{\prime}$ increases. So, there exists an optimum position of energy level $E^{\prime}{ }_{Q}$ leading to a maximum cooling rate $\dot{Q}_{C \max }$. As the width of energy level $\Delta E$ increases, the position of energy level $E_{Q}^{\prime}$ corresponding to the maximum cooling rate decreases, while the maximum cooling rate increases firstly and then decreases. For given $\Delta E$, as the position of the energy level $E^{\prime}$ approaches the special energy $E_{0}$, the cooling rate decreases and becomes negative. This is because refrigeration and heat pumping mix and counteract one another, or the cooling rate turns into a heating rate. The electrons with energies only in the ranges $\mu_{c}<E^{\prime}$ and $E^{\prime}+\Delta E<E_{0}$ contribute positively to the value of $\dot{Q}_{C}$.

Figure 3 shows that the COP increases firstly and then decreases as the position of energy level $E^{\prime}$ increases. There exists an optimum position of energy level $E_{\varepsilon}^{\prime}$ leading to a maximum $\mathrm{COP} \varepsilon_{\max }$. 


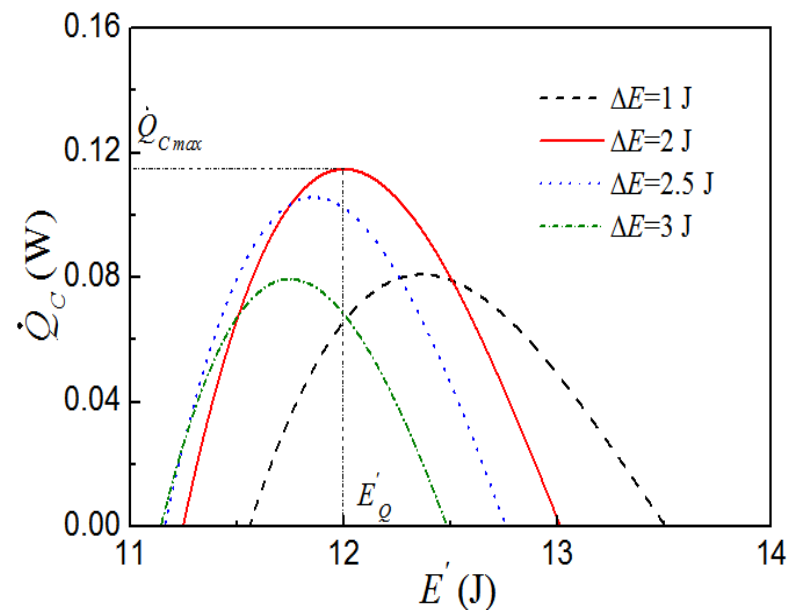

Figure 2. The variation of the cooling rate with respect to the position of energy level.

As the width of energy level $\Delta E$ increases, the position of energy level $E_{\varepsilon}^{\prime}$ corresponding to the maximum cooling rate decreases, and the maximum COP $\varepsilon_{\max }$ also decreases. Obviously, the maximum COP is lower than Carnot value, i.e. $\varepsilon_{\max }<\varepsilon_{c}=T_{C} /\left(T_{H}-T_{C}\right)=1$.

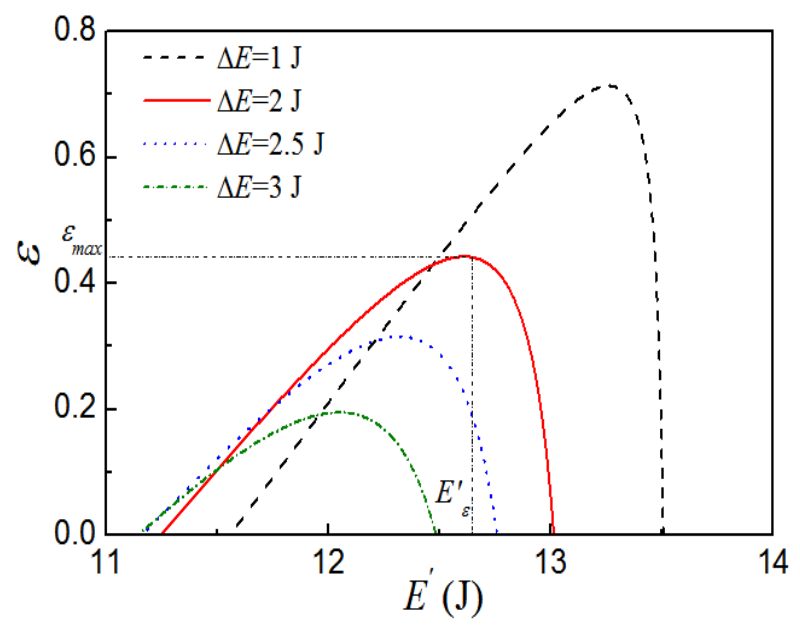

Figure 3. The variation of COP with respect to the position of energy level.

Especially, the curves of the cooling rate versus the COP are plotted in Figure 4. It is found that the performance characteristic curves between the cooling rate and the COP are the closed loop-shaped ones. The maximum cooling rate $\dot{Q}_{c \max }$ and the corresponding COP $\varepsilon_{Q}$, as well as the maximum COP $\varepsilon_{\max }$ and the corresponding cooling rate $\dot{Q}_{c \varepsilon}$ can be numerically calculated at the given parameters.

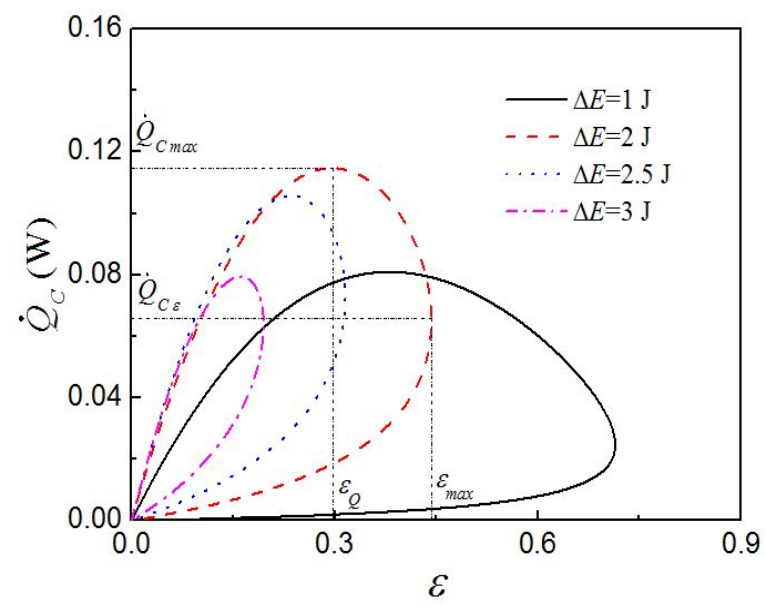

Figure 4. The characteristic curves between COP and $\dot{Q}_{C}$ at different values of $\Delta E$.

The optimal operating regions of the refrigerator should be located in those of the $\dot{Q}_{C} \sim \varepsilon$ curve with a negative slope. It is clearly shown that when the cooling rate is in this region, there are two different COPs for a given cooling rate, where one is smaller than $\varepsilon_{Q}$ and the other is larger than $\varepsilon_{Q}$. As a refrigerator, one always wants to obtain the COP as large as possible for a given cooling rate. Thus, the optimal COP of the refrigerator should be

$\varepsilon_{Q} \leq \varepsilon \leq \varepsilon_{\max }$

$\varepsilon_{Q}$ and $\varepsilon_{\text {max }}$ are two important performance parameters which determine the lower and upper bounds of the optimal COP. The cooling rate $\dot{Q}_{C}$ should be

$\dot{Q}_{C \varepsilon}<\dot{Q}_{C}<\dot{Q}_{C \max }$

$\dot{Q}_{C \varepsilon}$ and $\dot{Q}_{C \max }$ are also two important performance parameters which determine the lower and upper bounds of the optimal cooling rate.

\section{Maximum Figure of Merit Regime}

For a refrigerator, the wanted thing is that both the cooling rate and the COP are as large as possible simultaneously. But we have seen above that in the optimal region the larger the cooling rate is, the smaller the COP is, 
and vice versa. Thus, neither the cooling rate $\dot{Q}_{C}$ nor the COP is a good target function for determining an optimal regime of refrigeration. In order to pay equal attention to the cooling rate and the COP, one select

$$
\chi=\varepsilon \cdot \dot{Q}_{C}
$$

as the target function for optimization. It is also called as the figure of merit [34]. A reasonable compromise is achieved by optimizing the figure of merit. Yan and Chen [34] first derived the coefficient of performance for an endoreversible Carnot-type refrigerator with linear (Newtonian) finite heat transfer law, i.e. $\varepsilon_{C A}=1 / \sqrt{1-T_{c} / T_{h}}-1$, which is the counterpart of CurzonAhlborn (CA) efficiency for refrigerators. Similar choice was also employed in Refs. [35-37] which report on different approaches to define refrigeration regimes.

It is seen from Eqs. (7), (8) that the COP and the cooling rate are the functions of the dimensionless scaled energy $r_{C / H}$, the width of the energy level $\Delta E$ and the temperature of the reservoir $T_{C / H}$. Thus, the figure of merit is also the functions of $r_{C / H}, \Delta E$ and $T_{C / H}$, i.e.

$$
\chi=\chi\left(r_{C / H}, T_{C / H}, \Delta E\right)
$$

Using Eqs. (7), (8), (11) and the extremal condition

$$
\begin{aligned}
& \left(\frac{\partial \chi}{\partial r_{C}}\right)\left(r_{C}=r_{C \max \chi}, r_{H}=r_{H \max \chi}\right)=0 \\
& \left(\frac{\partial \chi}{\partial r_{H}}\right)\left(r_{C}=r_{C \max \chi}, r_{H}=r_{H \max \chi}\right)=0
\end{aligned}
$$

we can obtain numerically the dimensionless scaled energy $r_{C / H \max \chi}$ at maximum figure of merit by using Mathematica program. The ranges of the temperature ratio $\tau$ and the width of energy level $\Delta E$ are chosen at $0<\tau<1$ and $0<\Delta E<10$, respectively. The only parameter $T_{C}=1 \mathrm{~K}$ is given. Then the corresponding cooling rate $\dot{Q}_{C \chi}$ and the $\mathrm{COP} \varepsilon_{\chi}$ are determined by substituting $r_{C / H \max \chi}$ into Eqs. (7) and (8). The three-dimensional diagram of the COP $\varepsilon_{\chi}$ as functions of the width of the energy level $\Delta E$ and the temperature ratio $\tau$, and the curves of the COP $\varepsilon_{\chi}$ versus the temperature ratio $\tau$ at different widths of the energy level $\Delta E$ are plotted at given $T_{C}=1 \mathrm{~K}$ as shown in Figures 5 and 6.

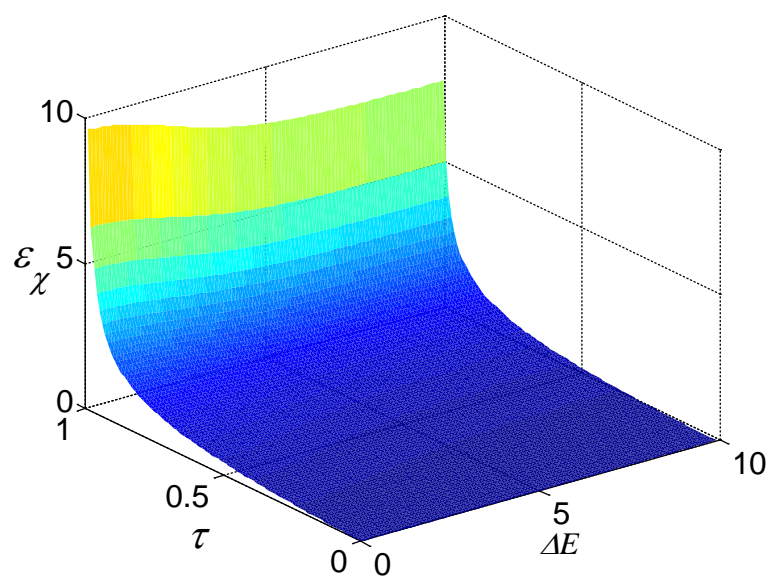

Figure 5. The three-dimensional diagram of the COP $\varepsilon_{\chi}$ as functions of the width of the energy level $\Delta E$ and the temperature ratio $\tau$ at given $T_{C}=1 \mathrm{~K}$.

It is seen from Figures 5 to 6 that the COP increases monotonously with increase of the temperature ratio $\tau$, while the COP decreases with increase of the width of energy level at given $\tau$. For given $\Delta E$, the closer the temperatures of two reservoirs are, the larger the COP at maximum figure of merit is. In comparison with the COP of endoreversible refrigerators $\varepsilon_{C A}=\frac{1}{\sqrt{1-\tau}}-1$, the COP is slightly higher than $\varepsilon_{C A}$ in the smaller width of energy level ( such as $\Delta E \rightarrow 0 \mathrm{~J}$ ), while the COP is lower than $\varepsilon_{C A}$ in the larger width of energy level (such as $\Delta E=2 \mathrm{~J}$ and $\Delta E=10 \mathrm{~J})$.

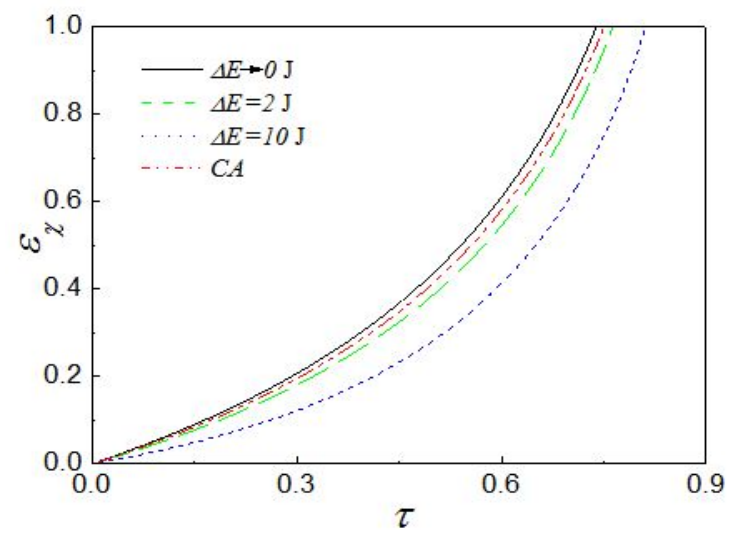

Figure 6. The curves of the COP $\varepsilon_{\chi}$ versus the temperature ratio $\tau$ at different widths of the energy level. 
The three-dimensional diagram of the cooling rate $\dot{Q}_{C \chi}$ are plotted as functions of the width of the energy level $\Delta E$ and the temperature ratio $\tau$, and the curves of the cooling rate $\dot{Q}_{C \chi}$ versus the temperature ratio $\tau$ at different widths of the energy level $\Delta E$ are plotted at given $T_{C}=1 \mathrm{~K}$ as shown in Figures 7 to 8.

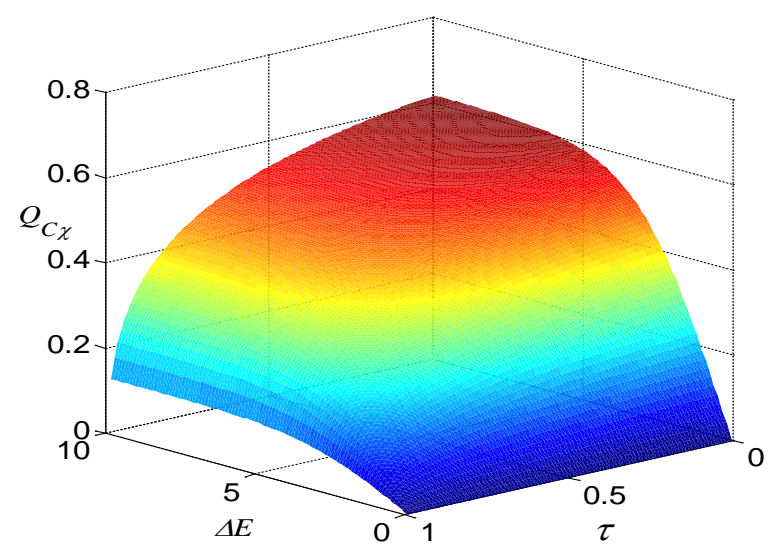

Figure 7. The three-dimensional diagram of the cooling rate $Q_{C \chi}\left(\dot{Q}_{C \chi}\right)$ as functions of the width of the energy level $\Delta E$ and the temperature ratio $\tau$ at given $T_{C}=1 \mathrm{~K}$.

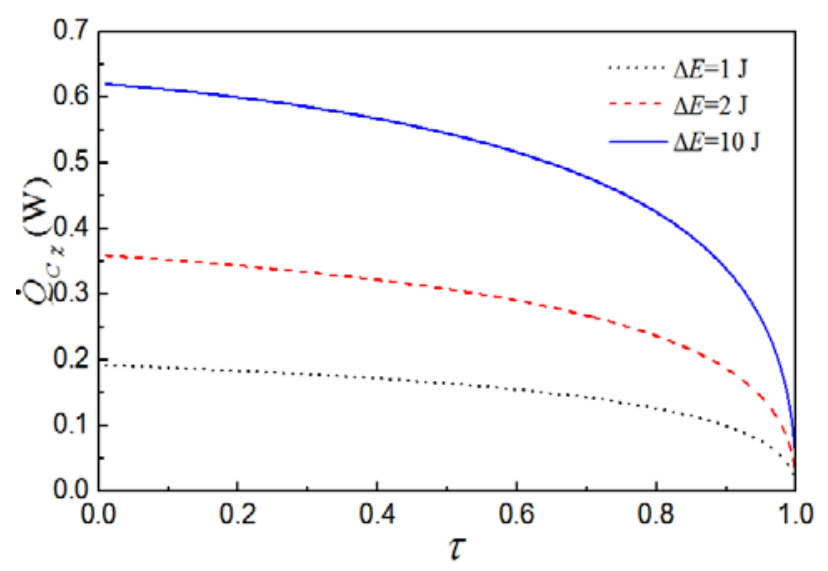

Figure 8. The curves of the cooling rate $\dot{Q}_{C \chi}$ versus the temperature ratio $\tau$ at different widths of the energy level.

It is seen from Figures 7 to 8 that the cooling rate $\dot{Q}_{C \chi}$ decreases monotonously with increase of temperature ratio $\tau$. At given $\tau$ the cooling rate increases with increase of the width of the energy level. The reason is the larger the width of the energy level is, the more the number of electrons via the energy level is. Thus, the larger the maximum cooling rate is. When $\Delta E \rightarrow 0$, the cooling rate $\dot{Q}_{C \chi}$ will vanish.

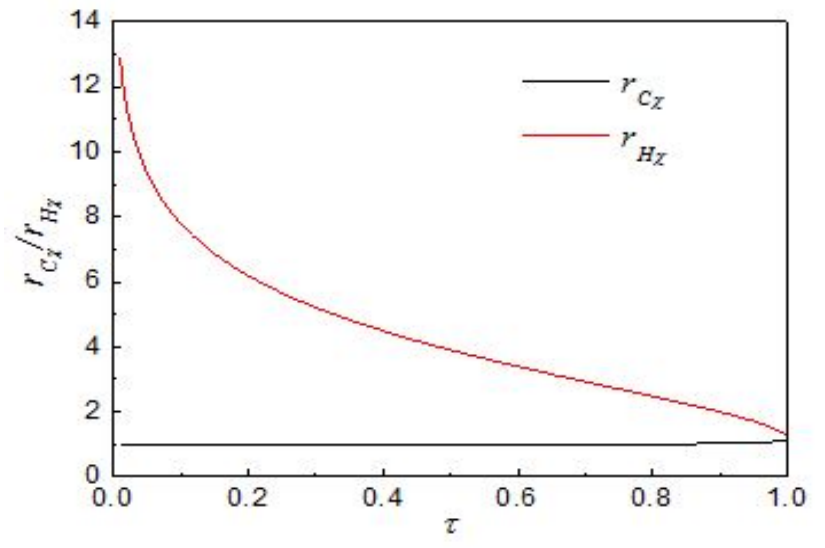

Figure 9. Dimensionless scaled energy $r_{H \chi}$ (upper) and $r_{C \chi}$ (lower) at maximum $\chi$ when $\Delta E=10 \mathrm{~J}$.

The curves of the dimensionless scaled energy $r_{H}$ and $r_{C}$ versus the temperature ratio $\tau$ is shown in Figure 9 at given $\Delta E=10 \mathrm{~J}$. The optimal ESE refrigerator performance $\chi$ obtain maximum, and the dimensionless scaled energy meet at $r_{C}=r_{H}=1.14543$. Actually, $r_{C / H}$ is function of $E^{\prime}-\mu_{C / H}$. According to the Figure 9 when $\tau$ fixed, giving the value of $\mu_{C / H}$, we can obtain the corresponding $E^{\prime}$.

\section{Conclusions}

A model of the ESE refrigerator with an ideal energy filter has been established in this paper. The analytical expressions for the cooling rate and the COP has been derived, respectively. The optimal regions of the cooling rate and the COP have been determined. Under the maximum figure of merit, the optimal performance characteristics of the ESE refrigerator has been analyzed. From the numerical results we found that: 1) the performance characteristic curve between the cooling rate and the COP is a closed loop-shaped one; 2) under the maximum figure of merit, the corresponding COP $\varepsilon_{\chi}$ increases and the cooling rate $\dot{Q}_{C \chi}$ decreases monotonously with increase of the temperature ratio $\tau$;3) at given temperature ratio $\tau$, the COP $\varepsilon_{\chi}$ decreases and the cooling rate increases with increase of the width of the energy level. The results obtained here can provide some theoretical guidelines for the design and operation of practical solid-state thermoelectric ESE refrigerators. 


\section{Acknowledgements:}

This work is supported by the National Natural Science Foundations of China (Grant Nos. 11065008 and 11365015) and the College Student Innovation Training Projects of Nanchang University (Grant No. 2012038).

\section{Nomenclature}

$E^{\prime} \quad$ position of energy level, $\mathrm{J}$

$e \quad$ charge of an electron, C

h Planck constant, $\mathrm{J} \cdot \mathrm{S}$

$k_{B} \quad$ Boltzmann constant, $\mathrm{J} / \mathrm{K}$

$P \quad$ power input, $\mathrm{W}$

$\dot{Q} \quad$ the rate of heat transfer, W

$T \quad$ temperature, $\mathrm{K}$

$V_{0} \quad$ bias voltage, $\mathrm{V}$

\section{Greek symbols}

$\varepsilon \quad$ coefficient of performance (COP)

$\mu \quad$ chemical potential, $\mathrm{J}$

$\chi \quad$ figure of merit, $\mathrm{W}$

$\tau \quad$ temperature ratio

\section{Subscripts}

C cold electron reservoir

$H \quad$ heat electron reservoir

max maximum

Q maximum cooling rate

$\varepsilon \quad$ maximum COP

$\chi \quad$ maximum figure of merit

\section{References}

[1] G. J. Snyder, E. S. Toberer, Complex thermoelectric materials, Nature Mater., 7, 105-114, 2008.

[2] P. Pichanusakorn, P. Bandaru, Nanostructured thermoelectrics, Mater. Sci. Eng. R, 67, 19-63, 2010.

[3] R. Ventkatasubramanian, E. Siivola, T. Colpitts, B. O’Quinn, Thin-film thermoelectric devices with high room-temperature figures of merit, Nature, 413, 597602, 2001.
[4] T. C. Harman, P. J. Taylor, M. P. Walsh, B. E. Laforge, Quantum Dot Superlattice Thermoelectric Materials and Devices, Science, 297, 2229-2232, 2002.

[5] K. F. Hsu, S. Loo, F. Guo, W. Chen, J. S. Dyck, C. Uher, T. K. E. Hogan, Cubic AgPbmSbTe2+m: Bulk thermoelectric Materials with High Figure of Merit, Science, 303, 818-21, 2004.

[6] N. Nakpathomkun, H. Q. Xu, H. Linke, Thermoelectric efficiency at maximum power in low-dimensional systems, Phys. Rev. B, 82, 235428, 2010.

[7] G. Min, D. M. Rowe, Experimental evaluation of prototype thermoelectric domestic-refrigerators, Appl. Energy, 83, 133-152, 2006.

[8] M. D. Ulrich, P. A. Barnes, C. B. Vining, Comparision of solid-state thermionic refrigeration with thermoelectric refrigeration, J. Appl. Phys., 90, 16251631, 2001.

[9] D. Vashaee, A. Shakouri, Improved thermoelectric Power Factor in Metal-Based Superlattices, Phys. Rev. Lett., 92, 106103, 2004.

[10] T. E. Humphrey, R. Newbury, R. P. Taylor, H. Linke, Reversible quantum Brownian heat engine, Phys. Rev. Lett., 89, 116801, 2002.

[11] T. E. Humphrey, Mesoscopic quantum ratchets and the thermodynamics of energy selective electron heat engines, Ph.D. Thesis , Sydney, Australia: University of New South Wales, 2003.

[12] T. E. Humphrey, H. Linke, Reversible thermoelectric nanomaterials, Phys. Rev. Lett., 94, 096601, 2005.

[13] M. F. O’Dwyer, T. E. Humphrey, H. Linke, Concept study for a high-efficiency nanowire based thermoelectric, Nanotechnology, 17, S1-6, 2006.

[14] M. F. O’Dwyer, R. A. Lewis, C. Zhang, Electronic efficiency in nanostructured thermionic and thermoelectric devices, Phys. Rev. B, 72, 205330, 2005

[15] B. X. He, J. Z. He, Thermoelectric refrigerator of a double-barrier InAs/InP nanowire heterostructure, Acta Phys. Sinica, 59, 3846-3850, 2010. ( in Chinese)

[16] B. X. He, J. Z. He, G. L. Miao, Influence of nanowire heterotructure on performance of electron refrigerator, Acta Phys. Sinica, 60, 040509, 2011. ( in chinese) 
[17] Z. Ding, L. Chen, F. Sun, Performance characteristic of energy selective electron (ESE) refrigerator with filter heat conduction, Rev. Mex. Fis., 56, 125-131, 2010.

[18] Z. Ding, L. G. Chen, F. Sun, Performance characteristic of energy selective electron (ESE) heat engine with filter heat conduction, I. J. Energy Eng., 2, 627-640, 2011.

[19] Z. Ding, L. Chen, F. Sun, Ecological optimization of a total momentum filtered energy selective electron (ESE) heat engine, Appl. Math. Modelling, 35, 276284, 2011.

[20] Z. Ding, L. Chen, F. Sun, Performance optimization of a total momentum filtered energy selective electron (ESE) heat engine with double resonance, Math. Comp. Model. Dyn. Syst., 54, 2064-2076, 2011.

[21] Z. Ding, L. Chen, F. Sun, Modeling and performance analysis of energy selective electron (ESE) engine with heat leakage and transmission probability, Sci. China; Phys. Mech. Astron., 54, 1925-1936, 2011.

[22] Z. Ding, L. Chen, F. Sun, Performance analysis of an irreversible energy selective electron (ESE) heat pump with heat leakage, J. Energy Inst., 85, 227-235, 2012.

[23] H. Wang, G. X. Wu, H. Lu, Performance of an energy selective electron refrigerator at maximum cooling rate, Phys. Scri., 83, 055801, 2011.

[24] H. Wang, G. X. Wu, Y. M. Fu, D. J. Chen, Performance analysis and parametric optimum criteria of the nanothermoeletric engine with a single-level quantum dot at maximum power, J. Appl. Phys., 111, 094318, 2012.

[25] J. Z. He, X. M. Wang, H. N. Liang, Optimum performance analysis of an energy selective electron refrigerator affected by heat leaks, Phys. Scri., 80, 035701, 2009.

[26] J. Z. He, B. X. He, Energy selective electron heat pump with transmission probability, Acta Phys. Sin., 59, 2345-2349, 2010.

[27] X. M. Wang, J. Z. He, J. Wang, Analysis of performance characteristics of the energy selective electron heat engine and refrigerator, Acta Electronica Sinica, 36, 2178-2182, 2008. (in Chinese)

[28] X. M. Wang, J. Z. He, W. Tang, Performance characteristics of an energy selective electron refrigerator with double resonances, Chin. Phys. B, 18, 984-990, 2009

[29] X. M. Wang, J. Z. He, H. N. Liang, Performance characteristics and optimal analysis of a nonlinear diode refrigerator, Chin. Phys. B, 20, 020503, 2011.

[30] J. Z. He, X. M. Wang, Y. C. Zhang, Performance characteristics of a quantum dot thermoelectric refrigerator, I. J. Mod. Phys. B, 26, 1250134, 2012.

[31] X. G. Luo, C. Li, N. Liu, R. W. Li, J. Z. He, T. Qiu, The impact of energy spectrum width in the energy selective electron low-temperature thermionic heat engine at maximum power, Phys. Lett. A, 377, 15661570, 2013

[32] L. Chen, Z. Ding, F. Sun, Model of a total momentum filtered energy selective electron heat pump affected by heat leakage and its performance characteristics, Energy, 36, 4011-4018, 2011.

[33] S. Su, J. Guo, G. Su, J. Chen, Performance optimum analysis and load matching of an energy selective electron heat engine, Energy, 44, 570-575, 2012.

[34] Z. Yan, J. Chen, A class of irreversible Carnot refrigeration cycles with a general eat transfer law, $J$. Phys. D: Appl. Phys., 23, 136-141, 1990.

[35] A. C. Hernandez, A. Medina, J. M. M. Roco, J. A. White, S. Velasco, Unified optimization criterion for energy converters, Phys. Rev. E, 63, 037102, 2001.

[36] C. D. Tomas, A. C. Hernandez, J. M. M. Roco, Optimal low symmetric dissipation Carnot engines and refrigerators, Phys. Rev. E, 85, 010104, 2012.

[37] Y. Wang, M. X. Li, Z. C. Tu, A. C. Hernandez, J. M. M. Roco, Cofficient of performance at maximum figure of merit and its bounds for low-dissipation Carnot-like refrigerators, Phys. Rev. E, 86, 011127, 2012. 